\title{
Haploidentical stem cell transplantation: a mini review
}

\begin{abstract}
Hematopoietic stem cell transplantation is arguably one of the most significant therapeutic measures initiated in the recent era, however this therapeutic entity from the outset was significantly limited by HLA matched donor availability. The advent of haploidentical stem cell transplantation with recently improving outcomes and reduced graft failure rates has provided the much needed boom to this aspect of modern day therapeutics by expanding the donor pool and reducing the wait time for a stem cell transplant which is prognostically important especially in aggressive leukemia's and lymphomas.
\end{abstract}

Keywords: hematopoietic stem cell transplantation, allogeneic haemopoietic stem cell, killer immunoglobulin like receptors, human leukocyte antigen
Volume 2 Issue 2 - 2017

\begin{abstract}
Akash Mathur,' Hemant Malhotra
'Department of Medicine, SMS Medical College Hospital, India 2Department of Medicine \& Head, Division of Medical Oncology, SMS Medical College Hospital, India
\end{abstract}

Correspondence: Hemant Malhotra, Senior Professor \& Head, Department of Medicine \& Head, Division of Medical Oncology, RK Birla Cancer Center, SMS Medical College Hospital, Jaipur 302004, India, Email drmalhotrahemant@gmail.com

Received: November 26, 2016 | Published: February 06, 2017
Abbreviations: HPC, allogeneic haemopoietic stem cell; HLA, human leukocyte antigen; MUD, matched unrelated donor; NK, natural killer; KIR, killer immunoglobulin like receptors; ATG, antilymphocyte globulin; PRS, post-relapse survival; URD, unrelated donors; UCB, umbilical cord blood

\section{Introduction}

Allogeneic haemopoietic stem cell (HPC) transplant involves matching a patient's human leukocyte antigen (HLA) tissue type with that of a related or unrelated donor. Advances in the medical field have now made possible the use of a haploidentical (partially matched) related donor for patients who are in need of a stem cell transplant but don't have a HLA-matched related or unrelated donor. Usually a haploidentical related donor is a $50 \%$ match to the recipient and can be the recipient's parent, sibling or any other relative. The benefit of having a haploidentical transplant is that it increases the probability of finding a donor as almost everyone will have a haploidentical donor. Initially haploidentical stem cell transplantation was limited by major complications like GVHD, rejection of the graft but with improvements in medical treatment, supportive care and pre/post transplant conditioning, the rate of such complications has significantly decreased to almost match that of transplants using HLA matched related or unrelated donors. Also, haploidentical SCT results in lower cost than cord or MUD (matched unrelated donor) transplants. Theoretically too there was this idea that the broad HLA disparity involved in haploidentical HSCT would result in a stronger graft versus leukemia effect in comparison with HLA matched transplants. ${ }^{1}$

\section{Selecting a haploidentical donor}

Amongst multiple available haploidentical donors the final choice is made based on certain donor aspects namely the age, gender, extent of HLA mismatch, anti donor antibodies, natural killer (NK) cell alloreactivity \& killer immunoglobulin like receptors (KIR) mismatch and non-inherited maternal antigen (NIMA) mismatch because donor selection can significantly impact the incidences of acute and chronic graft-versus-host disease, transplant-related mortality, and relapse, in addition to overall survival. ${ }^{2}$

\section{Modern day perspective: head to head with hla matched donor transplant}

Long $\mathrm{H}$ et $\mathrm{al}^{3}{ }^{3}$ while studying the long-term outcomes of HLA-haploidentical stem cell transplantation based on an FBCA (fludarabine, busulphan, cyclophosphamide, anti-thymocyte globulin) conditioning regimen compared with those of HLA-identical sibling stem cell transplantation for haematologic malignancies concluded that haplo-SCT could achieve nearly comparable outcomes to HLAidentical sibling SCT. In this study a comparison was conducted of 213 patients with haematologic malignancies who underwent HLA-identical sibling $(\mathrm{n}=108)$ or HLA-haploidentical $(\mathrm{n}=105)$ haematopoietic cell transplantation (haplo-HCT). The conditioning regimen included fludarabine, busulphan, cyclophosphamide and antilymphocyte globulin (ATG) (FBCA). The total dose of ATG differed between identical and haploidentical groups $(3.75 \mathrm{mg} /$ $\mathrm{kg}$ versus $12.5 \mathrm{mg} / \mathrm{kg}$ ). The cumulative incidences of grade II-IV acute GvHD in the identical and haploidentical groups were $20.4 \%$ and $21.9 \%(\mathrm{P}=0.73)$, and 2-year cumulative incidences of chronic GvHD were $36.4 \%$ and $24.1 \%(\mathrm{P}=0.17)$, respectively. The 3 -year probabilities of non-relapse mortality for identical and haploidentical groups were $20.5 \%$ and $34.9 \%(\mathrm{P}=0.048)$, and for relapse were $22.2 \%$ and $21.0 \%(\mathrm{P}=0.85)$, respectively. The 3 -year overall survivals in the identical and haploidentical groups were $62.6 \%$ and $52.6 \%(\mathrm{P}=0.054)$, whereas the 3 -year disease-free survivals were $54.7 \%$ and $43.1 \%$ $(\mathrm{P}=0.14)$, respectively. ${ }^{3}$

Similar findings were reiterated by Ma YR et al., ${ }^{4}$ while comparing post-relapse outcomes between haploidentical and matched related donor allogeneic stem cell transplantation. They investigated the impact of donor type on post-relapse survival (PRS) in 85 patients with hematological relapse after their first allogeneic hematological stem cell transplantation (allo-HSCT) for hematological malignancy. The median follow-up was 64 months among survivors. Both 3-year overall survival and 3-year PRS were similar in haploidentical donor (HID) and matched sibling donor (MRD) transplantation $(13.0 \% \pm 4.7 \%$ vs $19.4 \% \pm 7.1 \%, \mathrm{P}=0.913$ and $7.7 \pm 3.9 \%$ vs $9.7 \pm 5.3 \%$, $\mathrm{P}=0.667){ }^{4}$ 
Baker $\mathrm{M}$ et al..$^{5}$ compared Outcomes after Haploidentical or Unrelated Donor Bone Marrow or Blood Stem Cell Transplantation in adult Patients with Hematological Malignancies and supported the use of haploidentical transplantation with post-transplantation cyclophosphamide as an alternate transplantation technique for patients lacking HLA-matched sibling donors. In a series of 54 patients undergoing haploidentical donor transplantation (HAPLO) compared to those from a control group of patients receiving cells from matched or mismatched unrelated donors (URD) selected by diagnosis and stem cell source transplantation outcomes were compared. The grades II to IV acute GVHD (aGVHD) cumulative incidence at 6 months after transplantation did not differ much for these 2 groups $(63 \%$ for HAPLO and $53 \%$ for URD recipients; $\mathrm{P}=0.269$ ), neither did the cumulative incidence of aGVHD of severe grade III/IV (13\% for HAPLO and $8 \%$ for URD recipients; $\mathrm{P}=0.44){ }^{6}$

Yuqian Sun et al. ${ }^{6}$ also concluded in their retrospective study while studying unmanipulated haploidentical versus matched unrelated donor allogeneic stem cell transplantation in adult patients with acute myelogenous leukemia in first remission concluded that haploidentical transplantation may be an alternative to allogeneic transplantation using a fully matched 10/10 unrelated donor transplantation in patients with AML in first remission. ${ }^{7}$

The key points in the work done by Stefan O Ciurea comparing Haploidentical transplant with post-transplant cyclophosphamide versus matched unrelated donor transplant for acute myeloid leukemia reflected that GVHD after haploidentical transplant with post-transplant cyclophosphamide was lower as compared to HLAmatched unrelated donor transplant. Also there was comparable overall survival after haploidentical compared with matched unrelated donor transplant for AML.

\section{Graft failure : the barrier almost breached}

Off late there has been a significant improvement in the outcomes of allogeneic hematopoietic stem cell transplantation (AHSCT) using HLA-haploidentical related donors due to better bidirectional control of alloreactive reactions i.e. in both graft-versus-host and hostversus-graft directions. Better donor selection for patients in need of AHSCT based on our recognition of the role of humoral rejection in the occurrence of primary graft failure in this setting has widened the horizons of our understanding about causes of engraftment failure in these patients. Development of appropriate multilineage therapeutic measures for HLA sensitized patients, especially the use of ATG, has also gone a long way in preventing this dreaded complication which is usually associated with a substantial mortality rate. With the advent of these recent advances the graft failure rate in haploidentical transplantation has decreased to less than $5 \%{ }^{8,9}$

\section{The road ahead}

Van Besien K et al., ${ }^{9}$ in their study of Haploidentical (haplo)-cord transplantation combined infusion of an umbilical cord blood (UCB) unit with CD34-selected cells usually from human leukocyte antigen (HLA) mismatched donors and suggested that initial rapid count recovery from the haplo-hematopoietic progenitors, is gradually replaced by durable engraftment from UCB progenitors thus benefitting overall outcomes ${ }^{5}$ Hence haploidentical SCT can also be used as a bridge to improving outcomes in patients undergoing UCB SCT thus achieving the best of both the worlds.

\section{Conclusion}

In conclusion, we can say that allogenic hematopoietic stem cell transplantation has come a long way with Haploidentical SCT as donors are readily available for most patients, collection is cheaper and rates of graft failure are acceptable and are improving with each passing day. While the disease spectrum utilizing haploidentical stem cell transplantation as a therapeutic measure widens this technique of modern day therapeutics continue to fulfill the promises with better response rates and improved overall survival.

\section{Acknowledgements}

None.

\section{Conflict of interest}

The author declares no conflict of interest.

\section{References}

1. Aggarwal M, Agrawal N, Ahmad R, et al. Haploidentical stem cell transplant: Established treatment, expanding horizons. Asian J Oncol. 2016;2(1):8-13.

2. Chang Y, Luznik L, Fuchs E, et al. How do we choose the best donor for T-cell-replete, HLA-haploidentical transplantation? Journal of Hematology \& Oncology. 2016;9(1):35.

3. Long H, Lu ZG, Song CY, et al. Long-term outcomes of HLA-haploidentical stem cell transplantation based on an FBCA conditioning regimen compared with those of HLA-identical sibling stem cell transplantation for haematologic malignancies. Bone Marrow Transplant. 2016;51(11):1470-1475.

4. Ma YR, Xu LP, Zhang XH, et al. Comparable post-relapse outcomes between haploidentical and matched related donor allogeneic stem cell transplantation. Bone Marrow Transplant. 2017;52(3):409-414.

5. Baker M, Wang H, Rowley SD, et al. Comparative outcomes after haploidentical or unrelated donor bone marrow or blood stem cell transplantation in adult patients with hematological malignancies. Biol Blood Marrow Transplant. 2016;22(11):2047-2055.

6. Sun Y, Beohou E, Labopin M, et al. Unmanipulated haploidentical versus matched unrelated donor allogeneic stem cell transplantation in adult patients with acute myelogenous leukemia in first remission: a retrospective pair-matched comparative study of the Beijing approach with the EBMT database. Haematologica. 2016;101(8):e352-e354.

7. Bayraktar U, Lima M, Ciurea S. Advances in haploidentical stem cell transplantation. Revista Brasileira de Hematologia e Hemoterapia. 2011;33(3):237-241.

8. Chang YJ, Huang XJ. Improving the clinical outcome of unmanipulated haploidentical blood and marrow transplantation. Bone Marrow Transplant. 2015;50(Suppl 2):S21-S23.

9. Van Besien K, Childs R. Haploidentical cord transplantation-The best of both worlds. Seminars in Hematology. 2016;53(4):257-266. 